\title{
PEREGRINACION AL CENTRO: ESENCIA DEL. AXIS MUNDI EM FUENTEOVEJUNA
}

\author{
José A. Madrigal
}

Al título del presente estudio, "Peregrinación al centro: esencia del axis mundi en Fuenteovejuna", se le debería añadir aún otro subtitulo que ilustrara mejor no sólo la base metodológica que va a emplearse, sino también su indole general. Dicho subtítulo podría ser: "ensayo de fenomenología aplicada", o quizás uno más específico como: "ensayo de redución eidética." El vocablo "eidética", que proviene del griego eidos y significa idea, aqui ha de entenderse de acuerdo con la jerga fenomenológica, la cual le ha asignado el significado o la traducción de "esencia." En otras palabras, el presente estudio es un ensayo sobre la arqueologia de la esencia del axis mundi en Fuenteovejuna y la orientación que debe seguirse para llegar a ella.

Como resultado de esta orientación filosófica, el trabajo va a estar dividido en dos partes principales que, aunque idealmente deberian estar sincrónicamente desarrolladas, constituyen homogéneamente, no obstante, teoria y aplicación. En la primeira, se desarrollará de una forma bastante lacónica los fundamentos básicos de la fenomenología de Edmundo Husser|1 (1859-1938) quien, además de ser considerado como la figura más sobresaliente de dicho movimiento filosófico, se reconoce como su primer y mejor exponente. A la par, se tratará de evitar a toda costa la tautologia que parece caracterizar gran parte de los tratados filosóficos, incluyendo los del mismo Husserl. En la segunda división, el propósito fundamental circunvalará alrededor de la aplicación del método de reducción eidética a la obra literaria, y específicamente al fenómeno del centro o axis mundi, el cual se revela sincrónicamente, y casi isócronamente, en dos puntos isobáricos y microcósmicos que encarnan la esencia de la restauración de la armonía del cosmos socio-político, histórico y ontológico de España y sus habitantes. Susodichos puntos - Cuidad Real y la Plaza en Fuenteovejuna - constituyen, al parecer, los verdaderos polos magnéticos de la acción dramática que subyacen bajo los cuatro niveles que el profesor William McCrary ya ha señalado en su estudio sobre la obra. ${ }^{2}$ 
Indudablemente, una de las corrientes filosóficas más importantes, y probablemente la más fundamental en el desarrollo de la filosofia moderna, es la Fenomenologia: doctrina que se desarrolla como auténtico sistema filosófico hacia las postrimerías del siglo $\mathrm{XIX}$ y principios del $\mathrm{XX}$, bajo las rigurosas investigaciones del alemán Edmundo Husserl. ${ }^{3}$

El propósito de los próximos párrafos es el exponer las características esenciales, al igual que la metodología, de esta doctrina cuya base epistemológica constituye la descripción y la clasificación de los fenómenos o actos de percepión como vehiculo para llegar a las esencias inherentes en si mismos. Esta exegesis debe empezar con una pregunta básica: ?qué es una apariencia o fenómeno para Husserl? 1) una apariencia es algo de que estamos conscientes y 2) es la manifestación de una esencia de aquéllo que es la apariencia. Como puede verse, en la fenomenología de Husserl no sólo no puede disociarse la conciencia de lo percibido, sino tampoco el sentido del ser fenómeno, que es lo percibido, (Ideas, capítulo I).

Sin embargo, el mejor método par allegar a un entendimiento más perspicuo de estos puntos tan fundamentales, es el hacer una breve ilustración de indole comparada entre René Descartes (1596-1650) y Edmundo Husserl. El primero, como bien se sabe, dividió el universo en dos substancias independientes y exclusivas una de otra: el rescogitans (el yo pensante - la conciencia) y el res extensa (la extención o el mundo material). Tal división, cuyo punto de origen es la duda cartesiana, refleja la preocupación central de Descartes: si el yo, mi yo, es de lo único que puedo estar seguro, ?cómo puedo transitar de la existencia mia y de mis pensamientos a otras existencias? Consecuentemente, esta abertura, o bifurcación tan radical que él crea entre sujeto-objeto, constituye el quehacer fundamental de la Fenomenologia. 4 No es hasta Husserl, y ya después de haber rechazado la tendencia empírica del psicologismo, que se trata de rechazar la dicotomía mente-naturaleza, y a su vez explicar lógicamente su relación inherente. Para él, el error de Descartes fue fatal a la filosofía, y dió lugar no sólo a escuelas que ponian énfasis en el racionalismo o en el empirismo, sino que ya para el siglo XIX el factor subjetivo se ignoraba como impertimente, al igual que carente de valor filosófico. Uno de los resultados más visibles fue el tratar la conciencia como un fenómeno empírico que podía investigarse cuantitativamente. Aun la psicología, em proceso de transformación, evolucionaba 
hacia una ciiencia del comportamiento donde todo podía medirse y verificarse.

Por tanto, la encuesta de la Fenomenología es doble: 1) establecer que la conciencia no es un objeto entre muchos otros en la naturaleza y, por consiguiente, no puede ser determinada a través de métodos cuantitativos y 2) negar la bifurcación cartesiana entre res cogitans y res extensa.

El primer paso de la fenomenología de Edmund Husserl se encuentra resumido en su famosa frase: "hay que retornar a las cosas mismas." Las cosas, según él, son los fenómenos: y los fenómenos, a su vez, son cualquier contenido, ya sea empírico o a priori, de que la conciencia está consciente. Antes de continuar, quizás sea necesario aclarar que el propósito del trabajo no es investigar la esencia de la conciencia misma, lo cual constituiría un viaje por el campo de la fenomenología pura, sino la esencia del fenómeno. O sea, un estudio de fenomenologia aplicada.

En vista de lo dicho, lo cual debe haber demostrado claramente que la postura fenomenológica constituye una antítesis al solipsismo, debe pasarse a explicar los otros dos conceptos fundamentales que forman la base de su metodologia: la intencionalidad (Ideas, 107-11, 222-30) y la reducción (Ideas, 155-67).

El concepto de la intencionalidad, tomado de su maestro Franz Brentano (1838-1917)5, pero despojado de su índole puramente psicológica, viene a constituir la razón de ser de los actos de la conciencia ya que para él el principio de intencionalidad es lo que hace posible "que la conciencia siempre es consciencia de algo, [es decir] que sólo es conciencia si es conciencia dirigida hacia un objeto (sentido de intentio). A la par, el objeto no puede ser definido, sino en su relación con la conciencia [ya que] es siempre objeto para un sujeto" (Dartigues, 27). Por extensión, cabe decir "que las esencias no poseen ninguna existencia fuera del acto de conciencia que a ellas apunta" (Dartigues, 27), lo cual significa que dicho acto de intencionalidad no constituye una percepción de la realidad sensible, sino una correlación entre conciencia y esencia que es, en realidad, co-original. Al igual, seria conveniente en esta etapa del trabajo descartar la terminología sujeto-objeto, que sólo parece indicar o reflejar la dicotomía cartesiana ya superada por la Fenomenología, por la de noesis-noema: vocablos griegos que Husserl considera más apropiados para describir la correlación inherente en el acto intencional. Noesis, que 
significa percepción mental, pensamiento o inteligencia, lo denomina como la actividad de la conciencia; mientras noema, que expresa "eso que es percibido" o "algo que es pensado," lo denomina como la esencia de esa actividad mental. 6

El segundo concepto, igualmente necesario para poder comprender aún mejor el concepto de la intencionalidad, es el de la reducción. Ha de intercalar-se que Husserl no usa un solo término para describir dicha metodología, sino varios, entre los cuales se encuentran: reducción eidética; reducción fenomenológica; "época" fenomenológica, procedente del griego epoché (término técnico usado por la escuela de los escépticos griegos para referirse a la suspensión de un juicio u obstrución de una creencia); y "acorchetar" (processo que consiste en el empleo de corchetes, en un sentido matemático, para suspender la actitud natural del individuo que proviene de las asunciones naturalistas y psicológicas, al igual que de ciertas ideas comunes y culturales). ${ }^{7}$ No obstante, todos estos términos son básicamente sinónimos de un método de reducción, cuyo común denominador es el cambio de actitud necesario para llevar a cabo un procedimiento metódico, en el cual la persona se sitúa en una esfera transcendental donde puede percibir las cosas tales como son, independientes de ningún prejuicio o idea preconcebida. Desde este punto en adelante se usará únicamente el término reducción.

La segunda división del trabajo, dado el carácter teórico de la primera, se podrá concentrar en la aplicación del método fenomenológico a Fuenteovejuna 8 ; y específicamente a la trayectoria hacia la plaza y, parelelamente, a Ciudad Real. Igualmente, debe indicarse que el énfasis o hincapié de este estudio reside en la perspectiva del lector o del espectador de la obra al percibir y subsecuentemente reducir el fenómeno; y no en la de los personajes dramáticos, a pesar de que es a través de sus acciones que puede dilucidarse la esencia del centro o axis mundi.

En el curso de la acción dramática resaltan seis momentos claves que toman lugar en la plaza de Fuenteovejuna: 1) la discusión o diálogo platónico $(828-31)$; 2) la reunión para resolver la crisis del pósito (836-38); 3) la anunciación a Juan del futuro matrimonio entre Laurencia y Frondoso, y la consumación del mismo (824-43); 4) las deliberaciones de los villanos sobre qué medidas tomar contra el Comendador (845-47); 5) la 
celebración de la venganza (850-51); y 6) el castigo colectivo (851-53). Interesantemente, puede notarse que aunque estos seis instantes dramáticos ocurren en diferentes actos y los personajes varían, todos tienen un común denominador: el reflejar, de una forma u otra, el estado colectivo de Fuenteovejuna y España. Analicemos cada uno de ellos.

En el primero, más conocido por diálogo platónico, lo que sobresale a primera vista es la división tan fundamental que existe entre villanos. Tal fragmentación, de indole antitética al estado colectivo que se percibe al final de la obra, refleja microcósmicamente no sólo lo que ocurre en Fuenteovejuna, sino también en España ya que inmediatamente a este episodio, se anuncia la caida de Ciudad Real. Por consiguiente, el hecho de que los villanos no pueden concurrir en lo más básico-el amor como fuerza armónica - paralela la desunión en el nivel nacional. Al igual, si el propósito del primer acto es poner el caso, no es difícil ver que lo que acontece en la plaza tiene una función microcósmica.

El segundo momento, concomitante a la apertura del segundo acto, revela la gran crisis que se aproxima a consecuencia del insuficiente acopio de granos en el pósito, como resultado de la participación del Comendador en la guerra. En otras palabras, lo que afecta a España afecta a Fuenteovejuna y viceversa. Sin embargo, los aspectos más fundamentales de estas escenas son dos: 1) la peregrinación, ya sea consciente o inconsciente, a la plaza, al centro, para buscar soluciones a los problemas que se avecinan; y 2) la llegada del Comendador, elemento disociativo, que los echa de ella, previniendo que lleguen a tomar un acuerdo. Recuérdese que la primeira migración al centro, cuyo propósito fue el de solucionar el problema del amor, también termina, aunque no abruptamente, con la llegada del victorioso Fermán Gómez.

El tercer instante, coda de la transmutación espiritual de Laurencia, comprende el momento en que Esteban, Frondoso y ella se dirigen hacia la plaza para anunciar a Juan Rojo su decisión de matrimonio y, subsecuentemente, la consumación de éste. Esta prolepsis, que en realidad representa la catástasis de la acción, toma lugar, al contrario de los dos primeros casos, sin la interrupción del Comendador, quien al llegar, ya irremediablemente tarde, sólo logra interrumpir las festividades que se celebran en el campo. De igual significación es que la boda en la Plaza y la derrota en Ciudad Real - puntos isobá- 
ricos - ocurren sincrónicamente, hasta el punto de parecer movimientos isócronos.

El cuarto momento, concordante al inicio del último acto, escenifica la reunión de los villanos en la sala de concejo de Fuenteovejuna. Por primera vez, ya totalmente conscientes del caos que ha causado el Comendador en ambos niveles, deliberan todos con menos confusión y con más sentido de orientación. Es momentos después que entra Laurencia desmelenada, y los incita definitivamente a la rebelión. O sea, puede verse una vez más la gravitación hacia este punto axial donde se refleja apodicticamente toda acción cósmica.

Ahora bien, si la rebelión es justificada poética y legalmente, la transgresión que sobreviene, en forma de mofación al muerto, requiere una acto de retribución de similar índole. Este desafuero se hace patente al observar que acto seguido a la venganza colectiva, la primera acción de los villanos es la de volver a la plaza con la cabeza de Fernán Gómez en una lanza. Por tanto, esta quinta peregrinación a la plaza revela dos aspectos fundamentales: 1) la confirmación de la victoria 0 , quizás sería más apropriado decir, la penúltima etapa en la trayectoria hacia lo que el profesor McCrary denomina como "un nuevo orden" (McCrary, 185) y 2) una transgresión que requiere cierto acto de justicia punitiva para salvar o establecer permanentemente la frágil armonia que es esencial a este nuevo orden.

El sexto y último momento, sincrónico a la última fase de dicha trayectoria, consiste de dos eventos que simbolizan la conquista permanente del centro: uno individual y otro colectivo. Por primera vez no sólo Frondoso y Laurencia pueden permanecer en la plaza sin miedo del comendador, sino también expresándose un amor cristiano y desinteresado que a su vez refleja la armonía total. A la par, la escena del juez impartiendo el castigo coletivo, además de representar la retribución a los excesos cometidos, significa la unión de Fuenteovejuna: microcosmos de la unión de España. Acto seguido, como es de esperarse, la acción cambia a la conversación entre los Reyes Católicos donde Fernando anuncia que Castilla, o sea Ciudad Real, queda en paz. Simultáneamente, el único que falta por unirse, el Maestre, pide perdón por su culpa y ofrece sus servicios al reino.

Dado el carácter de la discusión sobre la plaza, la perspectiva de la parte final del trabajo se orientará hacia la apli- 
cación del método filosófico de Husserl a este fenómeno, alrededor del cual parece circunvalar toda acción representativa.

Establecido el paso inicial, o sea el estar consciente de la multiplicidad de percepciones, puede proseguir el lector a la reducción del fenómeno. Dicha exégesis fenomenológica, encuesta hacia la dilucidación de las esencias, debe incoar con una serie de preguntas básicas sobre lo percibido. Por ejemplo: ?Por qué la plaza y Ciudad Real tienen tanta importancia? ?Qué relación tienen en la temática de la obra? ?Por qué los villanos, cada vez que tienen que resolver una crisis, se reúnen en ella? ?Por qué el Comendador hace todo lo posible porque no pueden permanecer? $\mathrm{Y}$ especialmente la pregunta más primordial ?Por qué las acciones que ocurren en estos puntos reflejan microcósmicamente actitudes y consecuencian generales? Sin duda alguna, a la multiplicidad de preguntas que se pueden formular, siempre sobresale la importancia de estos puntos geográficos como lugares centrales. Concentrando la atención solamente al axis mundi más prepondernate de la acción dramática, la plaza, puede observarse que en ella se hallan la iglesia, el pósito, el ayuntamiento, la confradia, etc.! instituciones que, lógicamente, sirven como sitios de congregación a los habitantes de la villa. Sin embargo, aceptar esta explicación como final y conclusiva, sería caer en una causalidad ilusoria y antitética a la verdadera esencia pura y transcendente del fenómeno. Es más, esta descripción de la plaza sólo constituye lo que Husserl denominó como "la actitud natural"; o sea, como una forma de percepción cultural que tiene suspenderse para el individuo poder situarse, a través del proceso de reducción, en una esfera donde sea posible la percepción de las cosas tales como son e independientes de ningún prejuicio o idea preconcebida. En otras palabras, dicho juicio $o$ actitud natural, procedente de la idiosincrasia de una época o cultura específica, tiene que ser obstruido si la esencia del fenómeno va a poder desentrañarse.

Es sólo después de empezar a reducir, de separar lo cultural de la cosa misma, que va quedando un residuo, el cual hace a la persona formularse dos preguntas fundamentales $y$ primondiales: ?Por qué lo que ocurre en la plaza refleja actitudes colectivas, al igual que situaciones cósmica? y ?Por qué es tan difícil no sólo el camino al centro, sino también el permanecer en él? Sin duda alguna, la plaza y la peregrinación hacia ella van percibiéndose, paulatinamente, no como actos derivados, típicos de un proceso de aculturación, sino como actos originales, más afines a la indole intencional del proceso 
co-original noético-noemático. Consecuentemente, a través de dicha reducción o análisis intencional, la plaza deja de ser plaza y se convierte en un centro o punto axial que, además de poseer ciertas características singulares, debe tener validez en cualquier época o cultura. Concomitantemente, el arduo camino hacia ella se transforma en un acto que refleja las dificultades del hombre al querer alcanzar un nuevo estado, al igual que tener acceso permanente a un lugar bastante inaccesible. Por consiguiente, ya puede notarse manifiestamente que en esta etapa no se percibe lo empírico, lo visible, sino lo esencial que, a su vez, hace posible la mejor comprensión de la apariencia original. Sin embargo, el percibir la esencia del fenómeno no concluye la indagación; al contrario, sólo propicia el vehículo para seguir dilucidando la función específica del centro en Fuenteovejuna. Debe recalcarse en este punto del trabajo que lo expresado previamente es posible ya que el acto de conciencia que percibe el fenómeno no es pura interioridad, sino un proceso abierto y dirigido hacia algo. Por tanto, el acto de conciencia no constituye la relación más primaria, al contrario, constituye parte de la modalidad de la experiencia humana debido a que el mundo, antes de dicho acto cognoscitivo, aparece ya constituido. O sea, el conocimiento es una relación encontrada y no creada. Es por esta razón que tiene que seguirse investigando el fenómeno porque, como ya se mencionó, posee una intención común cósmica.

Para continuar esta exégesis sobre el concepto del axis mundi, hay que recurrir a las investigaciones de Mircea Eliade, fenomenólogo religioso, quien ha dedicado numerosos estudios a su análisis, 9 con el doble propósito de probar su existencia y también de demostrar su proyección en múltiples civilizaciones y épocas. Según Eliade, toda región habitada tiene un centro, el cual, debido a que constituye la zona de lo sagrado por excelencia, es donde puede experimentarse lo real en su totalidad (Mito, 25). Para ilustrar mejor la idea del centro, ya sea de índole geográfica en un principio o simbólica más tarde, sería conveniente presentar, como ejemplo, lo que relata la mitología cristiana en cuanto al lugar donde toma lugar la creación y la muerte de Adán y más tarde la muerte de Cristo. Según varias leyendas, estos tres eventos ocurrieron en el mismo lugar. En otras palabras, si el nacimiento y defunción de Adán, microcosmos del hombre, y la muerte de Cristo, acción redentora del género humano, van a poseer un valor cósmico tienen que haber estado localizados en un punto axial de emanación total. 10 
Consecuentemente, el camino que conduce al centro no puede ser fácil, sino más bien arduo y lleno de peligrosas circunvoluciones, tal como puede evidenciarse en el caso de los villanos de Fuenteovejuna. La causa de este fenómeno hay que buscarla en el hecho de que la peregrinación a la realidad absoluta constituye un rito del paso de lo profano a lo sagrado; de lo efímero y lo ilusorio a la realidad y la eternidad; de lo exterior a lo interior; de la multiplicidad a la unidad; del espacio - lo inespacial; al igual que de lo temporal a lo intemporal (Mito, 25-6). Expresado de otra forma, el acceso al axis mundi equivale a una consagración, a una iniciación donde puede lograrse una existencia real, duradera y paradisíaca (Mito, 26). Asimismo, este ascenso iniciático, que esencialmente es un paso del caos a la armonía, también refleja la repetición ritualista del acto de la Creación.

Como consecuencia de las acciones primigenias de dicha indole, la repetición o imitación de estos fenómenos, en épocas futuras, adquiere una esencia ritualista que, inevitablemente, se convierte en un arquetipo sujeto a condiciones históricas, políticas, sociales, etc. Sin embargo, sin perder el significado original ya que en su aplicación reside la clave para poder apreciar las acciones del mundo visible.

Para concluir, sólo baste recapitular que a través del proceso de reducción, el lector puede percibir la esencia de los fenómenos. En el caso específico de Fuenteovejuna, el desciframiento de la plaza y de la peregrinación hacia ella ayuda a dilucidar no sólo el contenido temático del drama, sino también esa estructura o realidad, ya constituida, que forma la base peremne de todas las acciones humanas significativas.

\section{NOTAS}

$1 \mathrm{La}$ obra fundamental donde se expone al contexto filosbfico del método fenomenológico de Edmund Husserl es Ideas: General Introduction to Pure Phenomenology (1913). Sin embargo, para poder llegar a una mejor comprensión de la importancia e significación de dicha obra, dentro del marco total de su filosofía, es vital hacer un breve recuento cronológico de los momentos claves de su evolución ideológica, previa a la publicación de Ideas... en 1913.

Husserl empieza su formación intelectual, al igual que muchos otros filosofos y en especial René Descartes, en el área de las matemáticas. Su primer libro, Filosofía de la aritmética (1891), constituye un estudio en el cual, muy típico de su momento histórico y cientifico, trata de explicar como la psícologia constituye la verdadera base de no sólo las matemáticas, sino también de la lógica. Las consecuencias de estas asunciones, además de ser desastrosas, gracias el violento ataque a su libro por el famoso matemático Arnold W. von Frege, también fueron, afortunadamente, el momento clave y decisivo cuando se e- 
fectúa el cambio de orientación que va a alejar a Husserl del "psicologismo", y sobre todo del positivismo científico tan arraigado en su época, hacia sus estudios sobre los fundamentos de la lógica. El resultado de estos investigaciones fue un segundo libro titulado Investigaciones lógieas (1901), donde ya rechaza rotundamente sus teorías psicológicas y acepta la lógica como punto de partida de todo inquirir racional. Llega a la conclusión, después de demostrarlo con repetidos ejemplos, que la lógica es una ciencia de índole normativa, mientras que la recién nacida psicología es una ciencia que no pasa de ser descriptiva y sumamente empirica.

Ya dado este paso decisivo, en que rechazaba la psicología como base de la logica y se da cuenta del peligro de caer en una forma de relativismo $\mathrm{y}$ nihilismo al seguir una base solamente empírica, Husserl está listo para enfrentarse a la polarización cartesiana con una actitud positiva. Esta ardua tarea comenzó con una serie de cinco conferencis que él las denominó con el título de La idea de la fenomenología y que, a su vez, constituyeron no sólo la fase gestadora, sino el mismo embrión de su fundamental y radical método fenomenologico.

La edición que se ha seleccionado es la siguiente: Ideas: General Introduction to Pure Phenomenology. New York: Collier McMillan Publishers, 1975. Las futuras referencias serán tomadas de esta edición y aparecerán documentadas en el texto del trabajo bajo: Ideas.

2 William C. McCrary. "Fuenteovejuna: Its Platonic Vision and Execution." SP, 5 8(1961), 179-92. Las futuras referencias aparecerán documentadas en el texto del trabajo bajo: McCrary. Este estudio, en mi opinión( es el más fundamental en la vasta critica sobre Fuenteovejuna.

3 El término "fenomenología", pesar de estar asociado intrínsecamente y dar el nombre a la doctrina de Husserl, se encuentra en uso ya en 1764, cuando el alemán Juan Enrique Lambert (1728-1777) lo emplea en su obra titulađa Nuevo 6rgano, con el propósito de denominar la teoría en que las apariencias son fundamentales para el conocimiento del mundo empírico. Dentro de la misma línea ideológica. Emmanuel Kant (1724-1804), quien habia sido corresponsal de Lambert, también adopta el vocablo en una carta a éste donde habla de la fenomenología como una disciplina propedéutica al estudio de la metafísica. A pesar de esto, sin embargo, su objetivo era sólo demostrar que el conocimiento teórico estaba limitado al nivel de los fenómenos y, por consiguiente, era imposible llegar a tener una comprensión del ser o de lo absoluto; o sea, de lo que él denominaba "las cosas en sí mismas."

Ahora bien, si Lambert y Kant usan el término esporádicamente y sin ningún propósito sitemático, Jorge G. Hegel (1770-1831) lo sitúa definitivamente dentro de la tradición y evolución de la filosofía mođerna al publicar en 1807 un trabajo titulado Fenomenología del espíritu. Para Hegel, al contrario de Kant, hay que bucar su significado en la perspectiva idealista de su método filosófico, la cual afirma la indole cognoscitiva de lo absoluto a través de la experiencia humana. En otras palabras, la fenomenologia, ya elevada a la categoría de etapa fundamental en la filosofia de la idea, se revela como una posibilidad que la potencia cognoscitiva puede descrifrar para llegar al conocimiento absoluto. No obstante, no es hasta los tradados de Husserl que la Fenomenologia pasa a constituir un sistema filosófico integral y autosuficiente.

Para una introducción más detalla sobre la trayectoria del término, véase: André Dartigues. La fenomenología. Barcelona: Editorial Herder, 1975. Págs. 9-13. Las futuras referencias aparecerán documentadas en el texto bajo: Darti- 
gues. También véase como referencia: Dagobert D. Runes. Dictionary of Philosophy. Totowa, New Jersey.: Littlefield, Adams and Co., 1962. Págs. 123-24, $158-60,163,231-34$.

4 Para una apreciación crítica, al igual que sumarizadora, véanse los siguientes estudios: Joseph J. Kockelmans. "What is Phenomenology? Some Fundamental Themes of Husserl's Phenomenology." .Phenomenology. Garden City, New York: Doubleday, 1967. Págs. 24-36. Las futuras referencias aparecerán bajo: Kockelmans; David Stewart and Algis Mickunas. Exploring Phenomenology. A guide to the field and its Literature. Chicago: American Library Association, 1974; Dartigues, 15-57.

5 Franz Brentano, quien es responsable de que Husserl use el concepto de la intencionalidad como un punto fundamental en su filosofía, habia tomado dicho concepto de Aristóteles, para quien, originalmente, significaba la incompleja orientación de la mente hacia un objeto el cual empieza a adquirir su existencia con dicho contacto.

6 La actividad o proceso noético-noemático no debe ser confundido con el psiquico. Para aclarar este punto, Husserl repite en numerosas ocasiones que no puede identificarse lo noético con el sujeto, ni lo noemático con el objeto. Al igual, hace hincapié que la actividad noética tampoco puede identificarse con la actividad psicológica ya que no trata de un proceso físico, sino del significado de dicha experiencia. 30-2.

7 Para las diferencias especificas entre estos términos véase: Kockelmans,

$8 \mathrm{La}$ edición de Fuenteovejuna que se ha seleccionado es la incluida en Obras escogidas. Teatro. Vol. I. Ed. Federico Carlos Sáinz de Robles. Madrid: Aguilar, 1969. Las futuras referencias aparecerán documentadas en el texto del trabajo por la página.

$9 \mathrm{~V}$ anse los siguientes estudios: El mito del eterno retorno. Madrid: Alianza Editorial, 1972. Págs. 20-34. Las futuras referencias aparecerán documentadas en el texto del trabajo bajo: Mito; Images and Symbols. New York: A search Book, 1969. Págs. 27-56; Tratado de historia de las religiones. México: Ediciones Era, 1972. Págs. 340-44.

10 Otra perspectiva del centro del cosmos es qué como punto axial constituye la zona de intersección entre las tres regiones cósmicas: el cielo, la tierra y el infierno (mundos superior, terrenal y subterráneo) (Mito 23-4). Como consecuencia de esta proximidad o este contacto, no es difícil ver como la escena en que Laurencia llega desmelenada a la reunión, e incita a los villanos a la venganza violenta contra el Comendador, representa una clara indicación del acto de transgresión colectiva que va a llevarse a cabo. Dicho acto, además de acercárlos a la zona subterránea o infernal, ha de requerir otro acto, también en el centro, de igual intensidad. Sin embargo, en este caso tiene que ser de indole punitiva si es que van a experimentar una existencia armónica, al igual que formar parte del nuevo orden. Esta retribución, por supuesto, se traduce en forma de castigo colectivo, el cual es impartido por el Rey, a través de la figura del juez.

\section{Auburn University}

Departament of Foreign Languages

Auburn - Alabama

USA 